\title{
Affine charge and the $k$-bounded Pieri rule
}

\author{
Jennifer Morse ${ }^{1} \|$ and Anne Schilling ${ }^{2} \|$ \\ ${ }^{1}$ Department of Mathematics, Drexel University, Philadelphia, PA 19104, U.S.A \\ ${ }^{2}$ Department of Mathematics, University of California, One Shields Avenue, Davis, CA 95616-8633, U.S.A.
}

\begin{abstract}
We provide a new description of the Pieri rule of the homology of the affine Grassmannian and an affine analogue of the charge statistics in terms of bounded partitions. This makes it possible to extend the formulation of the Kostka-Foulkes polynomials in terms of solvable lattice models by Nakayashiki and Yamada to the affine setting.

Résumé. Nous proposons une nouvelle description de la règle de Pieri de l'homologie de la variété Grassmannienne affine et un analogue affine de la statistique de charge en termes de partitions bornées. Il est ainsi possible d'étendre au cas affine la formulation due à Nakayashiki et Yamada des polynômes de Kostka-Foulkes en termes de modèles de réseaux résolubles.
\end{abstract}

Keywords: Charge statistic, Pieri rule, $k$-Schur functions, energy function, affine Schubert calculus

\section{Introduction}

Our study concerns an affine generalization of the Kostka-Foulkes polynomials. Kostka-Foulkes polynomials comprise a fascinating family of polynomials in a single parameter $t$ that arises in diverse contexts such as cohomology of Springer fibers [25, 6], representation theory of $G L_{n}\left(\mathbb{F}_{q}\right)$ [21, Ch. IV], affine Kazhdan-Lusztig theory [20], solvable lattice models and crystal base theory [23], the Bethe Ansatz and rigged configurations [8], and the study of subgroups of finite abelian groups [1]. They also encode the irreducible decomposition of the Garsia-Procesi graded $S_{n}$-modules [5].

In symmetric function theory, the Kostka-Foulkes polynomials appear as Schur transition coefficients with Macdonald's $P$-functions, defined by

$$
P_{\lambda}(x ; t)=\frac{1}{v_{\lambda}(t)} \sum_{w \in S_{n}} w\left(x^{\lambda} \prod_{i<j} \frac{x_{i}-t x_{j}}{x_{i}-x_{j}}\right),
$$

where $\lambda$ is a partition of length $\leq n$ (with allowable zero parts), $v_{\lambda}(t)=\prod_{j>0} \prod_{i=1}^{m_{j}} \frac{1-t^{i}}{1-t}$ for $m_{j}$ the multiplicity of $j$ in $\lambda$, and $S_{n}$ is the symmetric group on $n$ elements. The set $\left\{P_{\lambda}\right\}$ forms a basis of the

\footnotetext{
${ }^{\dagger}$ Partially supported by the NSF grant DMS-1301695.

$\ddagger$ Partially supported by the NSF grant OCI-1147247.

Emails: morsej@math.drexel.edu and anne@math.ucdavis.edu

1365-8050 (c) 2015 Discrete Mathematics and Theoretical Computer Science (DMTCS), Nancy, France
} 
ring of symmetric functions $\Lambda$ over $\mathbb{Q}(t)$ and $P_{\lambda}(x ; t)$ reduces to the monomial symmetric function $m_{\lambda}$ when $t=1$. Kostka-Foulkes polynomials are inscribed in its transition with the Schur basis:

$$
s_{\lambda}(x)=\sum_{\mu} K_{\lambda \mu}(t) P_{\mu}(x ; t) .
$$

Lascoux and Schützenberger [17, 1] proved that the Kostka-Foulkes polynomials can be expressed as the generating function of the set $\operatorname{SSYT}(\lambda, \mu)$ of semi-standard Young tableaux of shape $\lambda$ and content $\mu$ graded by the (non-negative integer) charge statistic

$$
K_{\lambda \mu}(t)=\sum_{T \in \operatorname{SSYT}(\lambda, \mu)} t^{\operatorname{charge}(T)} .
$$

Subsequently, Nakayashiki and Yamada [23] showed that the Kostka-Foulkes polynomials can instead be interpreted as the partition function of an exactly solvable lattice model in statistical mechanics. In particular, $K_{\lambda \mu}(t)$ is expressed in terms of tensor products of type $A$ Kirillov-Reshetikhin (KR) crystals graded by the energy function. KR crystals $B^{r, s}$ are labeled by $r \in\{1,2, \ldots, n\}$ and an integer $s \geq 1$. In type $A_{n}^{(1)}, B^{r, s}$ can be considered as the set of all semi-standard Young tableaux of rectangular shape $\left(s^{r}\right)$ over the alphabet $\{1,2, \ldots, n+1\}$. The classical crystal operators $\tilde{f}_{i}$ and $\tilde{e}_{i}$ for $1 \leq i \leq n$ are defined in terms of certain bracketing rules involving the letters $i$ and $i+1$. The affine Kashiwara operators $\tilde{e}_{0}$ and $\tilde{f}_{0}$ are defined as $\tilde{e}_{0}=\operatorname{pr}^{-1} \circ \tilde{e}_{1} \circ \mathrm{pr}$ and $\tilde{e}_{0}=\mathrm{pr}^{-1} \circ \tilde{e}_{1} \circ \mathrm{pr}$, where pr is the promotion operator. See for example [4].

For any partition $\mu=\left(\mu_{1}, \mu_{2}, \ldots, \mu_{N}\right)$, let $B^{\mu}=B^{1, \mu_{N}} \otimes \cdots \otimes B^{1, \mu_{1}}$ be a tensor product of single row KR crystals and let $E: B^{\mu} \rightarrow \mathbb{Z}$ be the (right) energy function (see for example [23, 19]). Here we use the opposite of Kashiwara's convention for tensor products of crystals. Since the elements of $B^{1, s}$ are single row-shaped tableaux, each factor $b_{N} \otimes \cdots \otimes b_{1} \in B^{\mu}$ can be associated (by RSK column-insertion) to a recording tableau; it is obtained by placing the letter $i$ into the boxes of shape $\left(b_{i} \ldots b_{1}\right) /$ shape $\left(b_{i-1} \ldots b_{1}\right)$. For highest weight vectors $b \in B^{\mu}$ (that is $\tilde{e}_{i}(b)=0$ for $i=$ $1,2, \ldots, n)$, this is equivalent to placing a letter $i$ in row $j$ of the recording tableau if $j$ occurs in $b_{i}$. It was proven in [23] that the energy function is equal to the charge of the recording tableau and consequently,

$$
K_{\lambda \mu}(t)=\sum_{\substack{b \in B^{\mu} \text {,weight }(b)=\lambda \\ \tilde{e}_{i}(b)=0 \text { for } i=1,2, \ldots, n}} t^{E(b)} .
$$

Example 1.1 For $\mu=(2,2,1)$, the highest weight crystal elements in $B^{1,1} \otimes B^{1,2} \otimes B^{1,2}$ are

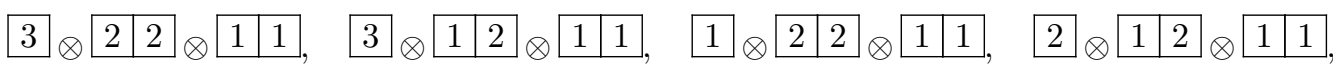

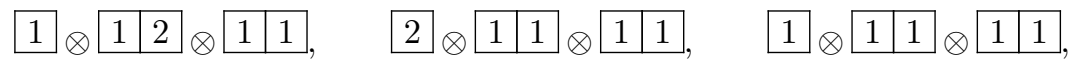

with energies $0,1,1,2,2,3,4$, respectively. Inserting the factors from right to left, recording the $i$-th factor with the letter $i$ yields the following corresponding tableaux:

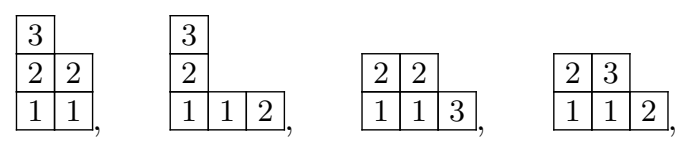




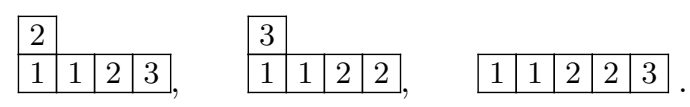

The charge of each recording tableau agrees with the energy of the corresponding tensor.

Note that the Kostka-Foulkes polynomials can also be related to tensor products of column KR crystals $B^{r, 1}[23,18]$.

Yet another direction ties Kostka-Foulkes polynomials to affine Schubert calculus. The algebraic nilHecke ring construction of Kostant and Kumar [7] and the work of Peterson [24] developed the study of Schubert bases associated to Schubert cells of the affine Grassmannian Gr for type $S L_{n}$ :

$$
\left\{\xi_{\lambda} \in H_{*}(\mathrm{Gr}, \mathbb{Z}) \mid \lambda \in \mathcal{P}^{n}\right\} \text { and }\left\{\xi^{\lambda} \in H^{*}(\mathrm{Gr}, \mathbb{Z}) \mid \lambda \in \mathcal{P}^{n}\right\},
$$

indexed by the subset $\mathcal{P}^{n}$ of partitions with all parts shorter than $n$. Separately, distinguished $k$-Schur and dual $k$-Schur bases for certain symmetric function spaces which are isomorphic to $H_{*}(\mathrm{Gr})$ and $H^{*}(\mathrm{Gr})$ were introduced and connected to Gromov-Witten invariants (quantum cohomology of Grassmannians) in [14, 15]. The approaches merged with Lam's proof [9] that these bases are sets of representatives for the Schubert classes of $H_{*}(\mathrm{Gr})$ and $H^{*}(\mathrm{Gr})$ (hereafter, $\left.k=n-1\right)$.

Recently, a $t$-generalization for the dual $k$-Schur basis was introduced in [3] using a construction in the spirit of (2) and (3). A non-negative statistic called kcharge is associated to certain chains in the Bruhat order on the type- $A$ affine Weyl group. The generating functions

$$
\mathcal{K}_{\lambda \mu}^{(k)}(t)=\sum_{T \in \mathcal{T}(\lambda, \mu)} t^{\mathrm{kcharge}(T)}
$$

are used to define polynomials, for $\lambda \in \mathcal{P}^{n}$, by

$$
\mathfrak{S}_{\lambda}(x ; t)=\sum_{\mu \in \mathcal{P}^{n}} \mathcal{K}_{\lambda \mu}^{(k)}(t) P_{\mu}(x ; t) .
$$

These form a basis for the subspace $\Lambda_{(n)}^{t}=\operatorname{span}\left\{P_{\lambda}(x ; t)\right\}_{\lambda_{1}<n}$ which reduces to a set of representatives for the Schubert cohomology classes $\left\{\xi^{\lambda}\right\}_{\lambda \in \mathcal{P}^{n}}$ when $t=1$.

To complement their geometric interpretation, our goal in this paper is to connect the affine KostkaFoulkes polynomials of (6) with Kirillov-Reshetikhin (KR) crystals. Our approach gives a new formulation for the Pieri rule for the homology of Gr in terms of partitions with restricted part sizes. From this, we use a description of kcharge given by Lapointe and Pinto in [16] in their study of the branching rules for $k$-Schur functions and also reformulate it in the $k$-bounded setting. In this setup, the Nakayashiki-Yamada correspondence with exactly solvable lattice models (or Kirillov-Reshetikhin crystals) is immediate. Another application of this new point of view is the pursuit of a combinatorial description of Gromov-Witten invariants ( $k$-Schur structure constants). The Schur structure constants can be described by counting skew tableaux with charge zero. The combinatorics presented here is in the same vein as classical tableaux combinatorics and should be useful in approaching the $k$-Schur constants.

\section{Affine Pieri rule}

The ring structure in homology $H_{*}(\mathrm{Gr})$ is determined by a refinement of the Pieri rule for Schur functions. The main ingredient used in [13, Section 9] to formulate the rule is an involution on $\mathcal{P}^{n}$ which refines 
partition conjugation. It is naturally defined through a bijection $\mathfrak{p}$ between $n$-cores (partitions that have no hooks of length $n$ ) and bounded partitions:

$$
\mathfrak{p}(\gamma)=\left(\lambda_{1}, \ldots, \lambda_{\ell}\right)
$$

where $\lambda_{i}$ is the number of cells with a $k$-bounded hook in row $i$ of an $n$-core $\gamma$. The $k$-conjugate of a shape $\lambda \in \mathcal{P}^{n}$ is then the shape

$$
\lambda^{\omega_{k}}=\mathfrak{p}\left(\mathfrak{p}^{-1}(\lambda)^{\prime}\right)
$$

where $\lambda^{\prime}$ denotes the conjugate of $\lambda$.

Theorem 2.1 [14] For any $\mu \in \mathcal{P}^{n}$, the affine Pieri rule is

$$
\xi_{m} \xi_{\mu}=\sum_{\lambda \in \mathcal{H}_{\mu, m}} \xi_{\lambda}
$$

where the sum is over the subset of the partitions, called weak horizontal m-strips, defined by

$$
\mathcal{H}_{\mu, m}=\left\{\lambda \mid \lambda / \mu=\text { horizontal m-strip and } \lambda^{\omega_{k}} / \mu^{\omega_{k}}=\text { vertical m-strip }\right\}
$$

Central to our study are coefficients arising in the following iteration of the affine Pieri relation:

$$
\xi_{\mu_{1}} \cdots \xi_{\mu_{\ell}}=\sum_{\lambda} \mathcal{K}_{\lambda \mu}^{(k)} \xi_{\lambda}
$$

These "affine Kostka coefficients" $\mathcal{K}_{\lambda \mu}^{(k)}$ count certain tableaux that are defined using $n$-cores and another notion from modular representation theory called the $n$-residue (the label $j-i \bmod n$ of a cell $(i, j)$ ).

Definition 2.2 Let $\gamma$ be a $(k+1)$-core with $m k$-bounded hooks and let $\alpha=\left(\alpha_{1}, \ldots, \alpha_{r}\right)$ be a composition of $m$. A "weak $k$-tableau" of shape $\gamma$ and weight $\alpha$ is a tableau of shape $\gamma$ with integers $1,2, \ldots, r$ such that the collection of cells filled with letter $i$ is labeled by exactly $\alpha_{i}$ distinct $(k+1)$-residues. Standard weak $k$-tableaux are those of weight $\left(1^{r}\right)$.

Note that for a weak $k$-tableau associated to a shape $\gamma$ and weight $\alpha$, in contrast to usual tableaux, $|\alpha|$ equals the number of $k$-bounded hooks in $\gamma$. We use $\mathcal{T}(\lambda, \mu)$ to denote the set of weak $k$-tableaux of weight $\mu$ and shape $\mathfrak{p}^{-1}(\lambda)$. This given, $\mathcal{K}_{\lambda \mu}^{(k)}=|\mathcal{T}(\lambda, \mu)|$.

The first step towards expressing affine Kostka polynomials in terms of KR crystals is to identify which recording tableaux of highest weight crystal elements correspond to weak $k$-tableaux. To do so, we reinterpret the Pieri rule for $\xi_{\lambda}$ in the context of a distinguished subset of semi-standard tableaux that gives yet another realization of weak $k$-tableaux.

Consider a semi-standard tableau $T$ of shape $\lambda$ and weight $\mu$. Denote by $\lambda^{(i)}$ the shape of the subtableau consisting only of the letters less than or equal to $i$. By 9 ,,$T$ is a $k$-bounded weak $k$-tableau if both

$$
\lambda^{(i)} / \lambda^{(i-1)} \text { and }\left(\lambda^{(i) \omega_{k}}\right)^{\prime} /\left(\lambda^{(i-1) \omega_{k}}\right)^{\prime} \quad \text { are horizontal strips for all } i \geq 1 .
$$

We have discovered a new characterization for $k$-bounded weak $k$-tableaux that employs an alternate formulation of $k$-conjugation due to Karola Mészáros [22]. 
Lemma 2.3 [22] [11] Chapter 2, Section 1.3]

1. Each row of a $k$-bounded partition $\lambda=\left(\lambda_{1}, \lambda_{2}, \ldots, \lambda_{\ell}\right) \in \mathcal{P}^{k+1}$ is assigned to a string as follows: the first string $s^{1}=\left(\lambda_{i_{1}}, \lambda_{i_{2}}, \ldots, \lambda_{i_{t}}\right)$ is constructed starting with the longest part $\lambda_{i_{1}}=\lambda_{1}$ and assigning other rows $\lambda_{i_{j}}$ satisfying $i_{j}=k+1-\lambda_{i_{j-1}}+i_{j-1}$. Successively construct strings $s^{i}$ for $i>1$, each time beginning with the longest row $\lambda_{i_{1}}$ that was not previously assigned to a string. Then

$$
\lambda^{\omega_{k}}=\left(\left|s^{1}\right|,\left|s^{2}\right|, \ldots,\left|s^{\ell}\right|\right)^{\prime} .
$$

2. The $i$-th part of $\mathfrak{p}^{-1}(\lambda)$ is obtained by adding all elements in the string containing $\lambda_{i}$ that are weakly above $\lambda_{i}$.

Example 2.4 For $\lambda=(4,3,3,2,1,1)$ and $k=4$, rows are assigned to the strings

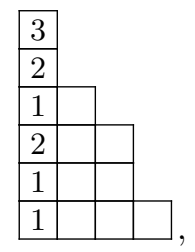

where the first cell in a row is labeled by its string, and

$$
\begin{aligned}
\left(\lambda^{\omega_{4}}\right)^{\prime} & =\left(\lambda_{1}+\lambda_{2}+\lambda_{4}, \lambda_{3}+\lambda_{5}, \lambda_{6}\right)=(9,4,1), \\
\mathfrak{p}^{-1}(\lambda) & =\left(\lambda_{1}+\lambda_{2}+\lambda_{4}, \lambda_{2}+\lambda_{4}, \lambda_{3}+\lambda_{5}, \lambda_{4}, \lambda_{5}, \lambda_{6}\right)=(9,5,4,2,1,1) .
\end{aligned}
$$

Remark 2.5 Given a partition $\gamma$ where $\gamma_{i}<\gamma_{i-1}$, let $\left(\gamma_{i_{1}}, \ldots, \gamma_{i_{t}}\right)$ denote the string to which $\gamma_{i}$ belongs. By construction, $\gamma_{i_{j}-1}$ is the first entry in its string for the smallest index $i_{j}$ larger than $i$.

We reformulate the conditions in (11) in terms of the strings defined in Lemma 2.3

Lemma 2.6 Let $\lambda, \tau \in \mathcal{P}^{k+1}$ such that $\lambda / \tau$ consists of one box in row $r$. Consider the string $s^{j}$ to which $\tau_{r}$ is assigned and let $s_{>r}^{j}=\left(\tau_{r_{1}}, \tau_{r_{2}}, \ldots\right)$ be the subset of parts in $s^{j}$ where $r_{1}>r$. Here we include parts of size 0 . Then $\left(\lambda^{\omega_{k}}\right)^{\prime} /\left(\tau^{\omega_{k}}\right)^{\prime}$ is a skew partition if and only if $\tau_{r_{i}}=\tau_{r_{i}-1}$ for all $i \geq 1$.

Proof: Let $s^{1}, s^{2}, \ldots$ and $b^{1}, b^{2}, \ldots$ denote the strings of $\tau$ and $\lambda$, respectively. Consider the string to which $\tau_{r}$ belongs; $s^{j}=\left(\tau_{i_{1}}, \ldots, \tau_{i_{a}}, \tau_{r_{1}}, \tau_{r_{2}}, \ldots\right)$ where $r_{1}>r=i_{a}$. Let $s^{t}$ denote the string to which $\tau_{r_{1}-1}$ belongs. Note by Remark 2.5 that $s^{t}=\left(\tau_{r_{1}-1}, \tau_{r_{2}^{\prime}}, \ldots\right)$. Since $\lambda$ differs from $\tau$ by only an additional box in row $r, s^{i}=b^{i}$ for all $i \neq t, j$. The additional box causes $r_{1}=k+1-\tau_{r}+r=(k+1-$ $\left.\lambda_{r}+r\right)+1$ and thus implies that the string to which $\lambda_{r}$ belongs is $b^{j}=\left(\tau_{i_{1}}, \ldots, \tau_{i_{a}}+1, \tau_{r_{1}-1}, \tau_{r_{2}^{\prime}}, \ldots\right)$ while $b^{t}=\left(\tau_{r_{1}}, \tau_{r_{2}}, \ldots,\right)$. Note by the string construction that $\tau_{r_{i}} \leq \tau_{r_{i}^{\prime}}$ implying that $\left|s^{j}\right|<\left|b^{j}\right|$ but $\left|s^{t}\right| \geq\left|b^{t}\right|$.

By Lemma 2.3 (1), it suffices to show that $\left|s^{i}\right| \leq\left|b^{i}\right|$ if and only if $\tau_{r_{i}}=\tau_{r_{i}-1}$, for all $i \geq 1$. Note that $\left|s^{i}\right| \leq\left|b^{i}\right|$ for all $i$ implies that $\left|s^{t}\right|=\left|b^{t}\right|$. In turn, this ensures that $\tau_{r_{i}}=\tau_{r_{i}^{\prime}}$ for all $i$. In particular, $\tau_{r_{i}}=\tau_{r_{i}-1}$. On the other hand, given $\tau_{r_{i}}=\tau_{r_{i}-1}$ for all $i, \tau_{r_{i}^{\prime}}=\tau_{r_{i}-1}$ and the claim follows. 
Example 2.7 Take $k=5$ and the partition $\tau$ with the strings as indicated

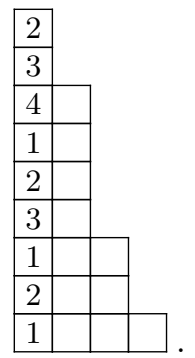

Then adding a box to row 2 of $\tau$ yields $\lambda$ such that $\left(\lambda^{\omega_{k}}\right)^{\prime} /\left(\tau^{\omega_{k}}\right)^{\prime}$ is a valid skew partition since $\tau_{4}=\tau_{5}$ and $\tau_{8}=\tau_{9}$, but adding a box in row 4 would not be allowed since $\tau_{8} \neq \tau_{7}$.

Let $\lambda, \tau \in \mathcal{P}^{k+1}$ such that $\lambda / \tau$ is a horizontal strip. Suppose that for a given row $r$ the skew partition $\lambda / \tau$ contains $\ell$ boxes, set $r_{1}=r+k+1-\tau_{r}$ (that is, the smallest row in $\tau$ above row $r$ in the same string as row $r)$, and let $s=\left(\lambda_{r_{1}}, \lambda_{r_{2}}, \ldots\right)$ be the tuple of parts of $\lambda$ weakly above row $r_{1}$ that belong to the string of Lemma 2.3 passing through row $r_{1}$. Again we include parts of size 0 .

Lemma 2.8 With the notation above, $\left(\lambda^{\omega_{k}}\right)^{\prime} /\left(\tau^{\omega_{k}}\right)^{\prime}$ is a horizontal strip if and only if $\lambda_{r_{i}}=\tau_{r_{i}-\ell}$ for all $i \geq 1$ and all rows $r$ with $\ell>0$.

Proof: The lemma follows from Lemma 2.6 by successively adding boxes. Adding boxes in different strings in $\tau$ is independent. Boxes within the same string need to be added from top to bottom, left to right.

Example 2.9 Take $k=5$ and

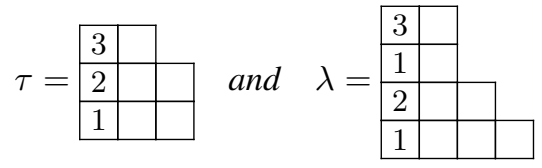

so that $\lambda / \tau$ has cells in rows 1 and 4 . Note that for row 1 , we have $r_{1}=4, \ell=1$ and $2=\lambda_{r_{1}}=\tau_{r_{1}-\ell \text {. }}$ Similarly, for row 4 we have $r_{1}=10, \ell=2$ and $0=\lambda_{r_{1}}=\tau_{r_{1}-\ell}$. Hence $\lambda / \tau$ is a weak horizontal strip for $k=5$.

On the other hand, for

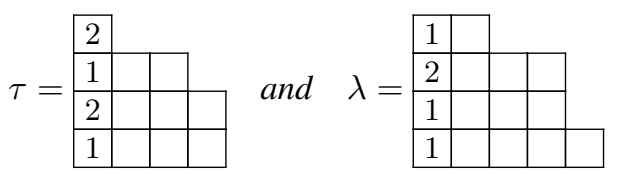

we have $\ell=1$ cells in row 1 of $\lambda / \tau$ and $r_{1}=3$, but $0=\lambda_{5}=\lambda_{r_{2}} \neq \tau_{r_{2}-\ell}=\tau_{4}=1$. Hence $\lambda / \tau$ is not a weak horizontal strip for $k=5$.

Given the Pieri rule Theorem 2.1, we may deduce a different formulation. 
Theorem 2.10 Let $1 \leq m<n$ and $\tau \in \mathcal{P}^{n}$. The Pieri rule in $H_{*}(\mathrm{Gr})$ can be described by

$$
\xi_{m} \xi_{\tau}=\sum_{\lambda} \xi_{\lambda}
$$

over all partitions $\lambda \in \mathcal{P}^{n}$ where $\lambda / \tau$ is a horizontal $m$-strip and $\lambda_{r_{i}}=\tau_{r_{i}-\ell}$ for all $i \geq 1$ and all rows $r$ in $\lambda / \tau$ with $\ell>0$ boxes, where $r_{i}$ is as defined in Lemma 2.8 .

Theorem 2.11 Let $T$ be a semi-standard tableau of $k$-bounded shape $\lambda$ and weight $\mu$. Then $T$ is a $k$ bounded weak $k$-tableau if for each letter $i$ in $T$, the skew shape $\lambda^{(i)} / \lambda^{(i-1)}$ satisfies the conditions of Lemma 2.8

Proof: This follows directly from Lemma 2.8 by noting that for a semi-standard tableau $T$ of $k$-bounded shape, condition (11) must hold for every $\lambda^{(i)} / \lambda^{(i-1)}$ in order to correspond to a weak $k$-tableau.

As a corollary of Theorem 2.11 we can now determine which highest weight crystal elements contribute to a $k$-Schur expansion of the Hall-Littlewood polynomials. Namely, it will be those whose insertion tableau is a $k$-bounded weak $k$-tableau by the conditions in Theorem 2.11. This, together with the $k$ charge in this language, is summarized in Theorem 3.8 .

Example 2.12 Let us determine which insertion tableaux from Example 1.1 contribute for $k=3$. Since the shape needs to be 3-bounded only the first four tableaux in (5) can potentially contribute. Looking at the strings in $\lambda^{(i)} / \lambda^{(i-1)}$ for each $i=1,2,3$ we find that
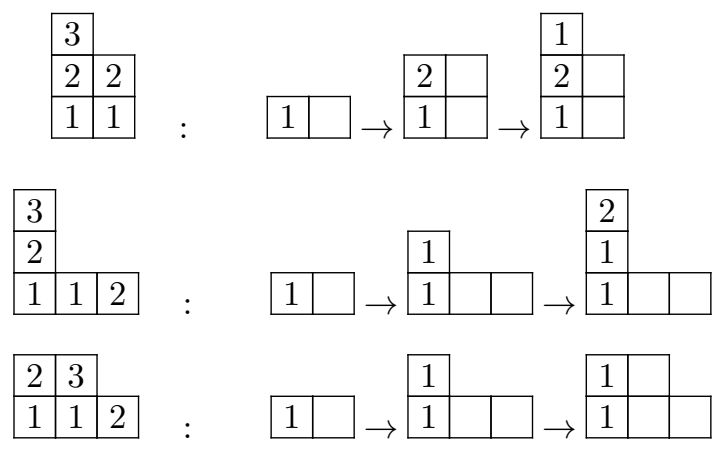

satisfy the conditions of Lemma 2.8 and hence contribute. On the other hand

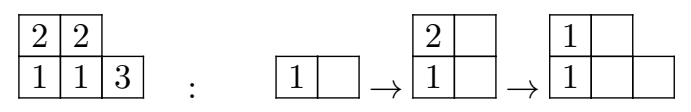

does not satisfy the condition of Lemma 2.8 for $\lambda^{(3)} / \lambda^{(2)}$ since in this case $\tau=(2,2), r=1, \ell=1$, $r_{1}=3$, and $0=\lambda_{3} \neq \tau_{2}=2$.

Since the shapes of the surviving tableaux are $(2,2,1),(3,1,1)$, and $(3,2)$, this agrees with the terms in the 3-Schur expansion in 16. How to obtain the powers of t is discussed in the next section. 


\section{Affine charge}

In [16], Lapointe and Pinto introduced a statistic called $k$-charge on weak $k$-tableaux. In analogy to (3), the affine Kostka numbers are thus lifted to affine Kostka polynomials

$$
\mathcal{K}_{\lambda \mu}^{(k)}(t)=\sum_{T \in \mathcal{T}(\lambda, \mu)} t^{\mathrm{kcharge}(T)} .
$$

It was recently proven [2] that the functions $\mathfrak{S}_{\lambda}(x ; t)$ can be defined in these terms.

The $k$-charge statistic is easiest to describe first on standard (of weight $\left(1^{n}\right)$ ) weak $k$-tableaux. Let $\operatorname{diag}\left(c_{1}, c_{2}\right)$, associated to cells $c_{1}$ and $c_{2}$ in a $(k+1)$-core, be the number of diagonals of residue $x$ that are strictly between $c_{1}$ and $c_{2}$ where $x$ is the residue of the lower cell. Instead of taking a cell as input, the diag function can also take a specific instance of an entry in the tableau as input (which then determines a cell).

Definition 3.1 Given a standard weak $k$-tableau $T$ on $m$ letters, put a bar on the topmost occurrence of letter $r$, for each $r=1, \ldots, m$. Define the index of $T$, starting from $I_{1}=0$, by

$$
I_{r}= \begin{cases}I_{r-1}+1+\operatorname{diag}(\bar{r}, \overline{r-1}) & \text { if } \bar{r} \text { is east of } \overline{r-1} \\ I_{r-1}-\operatorname{diag}(\bar{r}, \overline{r-1}) & \text { otherwise }\end{cases}
$$

for $r=2, \ldots, m$. The $k$-charge of $T$ is the sum of entries in $I(T)$, denoted by kcharge $(T)$.

The definition of $k$-charge extends to semi-standard weak $k$-tableaux by successively computing an appropriate choice of standard sequences. The trick is to introduce a method for making this choice in weak $k$-tableaux.

Definition 3.2 From an $x$ (of some residue $i$ ) in a semi-standard weak $k$-tableau $T$, the appropriate choice of $x+1$ will be determined by choosing its residue from the set A of all $(k+1)$-residues labelling $x+1$ 's. Reading counter-clockwise from $i$, this choice is the closest $j \in A$ on a circle labelled clockwise with $0,1, \ldots, k$.

A standard subtableau is extracted by choosing the rightmost $x=1$ and then selecting subsequent letters $x$ using the choice of Definition 3.2. The same procedure is then repeated with the yet unselected letters.

Example 3.3 For $k=3$, we compute the $k$-charge:

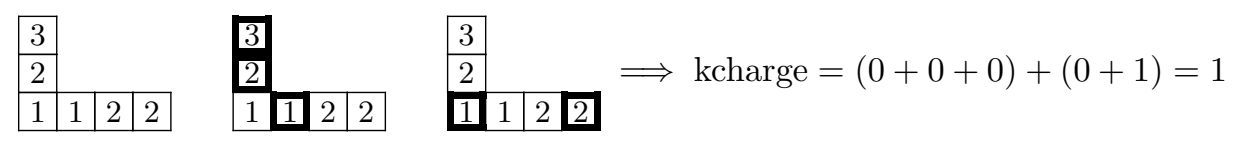

Hall-Littlewood polynomials $\left\{H_{\mu}(x ; t)\right\}$ arise as the basis dual to $\left\{P_{\mu}(x ; t)\right\}$ with respect to the Hall inner product

$$
\left\langle m_{\lambda}, h_{\mu}\right\rangle=\delta_{\lambda \mu}
$$

where $m_{\lambda}$ are the monomial symmetric functions and $h_{\lambda}$ are the complete homogeneous symmetric functions. Hence, (7) implies that

$$
H_{\mu}(x ; t)=\sum_{\lambda} \mathcal{K}_{\lambda \mu}^{(k)}(t) s_{\lambda}^{(k)}(x ; t)
$$


where $\left\{s_{\lambda}^{(k)}(x ; t)\right\}_{\lambda \in \mathcal{P}^{n}}$ is the dual basis to $\left\{\mathfrak{S}_{\lambda}(x ; t)\right\}_{\lambda \in \mathcal{P}^{n}}$. At $t=1, s_{\lambda}^{(k)}(x ; t)$ reduce to the parameterless $k$-Schur functions (discussed in the introduction).

Example 3.4 We have

$$
\begin{aligned}
H_{221}[X ; t] & =s_{221}^{(2)}=s_{221}^{(3)}+t s_{311}^{(3)}+t^{2} s_{32}^{(3)} \\
& =s_{221}^{(4)}+t s_{311}^{(4)}+\left(t+t^{2}\right) s_{32}^{(4)}+t^{3} s_{41}^{(4)} \\
& =s_{221}^{(5)}+t s_{311}^{(5)}+\left(t+t^{2}\right) s_{32}^{(5)}+\left(t^{2}+t^{3}\right) s_{41}^{(5)}+t^{4} s_{5}^{(5)}
\end{aligned}
$$

where we have dropped the dependence on $x$ and $t$ in the notation. For example, the coefficient of $s_{311}^{(3)}$ in 16 is $\mathcal{K}_{(3,1,1),(2,2,1)}^{(3)}(t)=t$ corresponding to the unique weak 3 -tableau of weight $\mu=(2,2,1)$ in Example 3.3 with kcharge equal to 1.

The $k$-charge in terms of $(k+1)$-cores as given in Definitions 3.1 and 3.2 can be reformulated for $k$-bounded weak $k$-tableaux.

Given a $k$-bounded weak $k$-tableau $T$ as characterized by Theorem 2.11, recall that $\lambda^{(i)}$ denotes the shape of $T$ restricted to the letters smaller or equal to $i$. Draw all intermediate shapes $\lambda^{(i)}$ together with their corresponding strings. Then for the new boxes in each step, compute the residues using $\mathfrak{p}^{-1}(\lambda)$ as described in Lemma 2.3 (2). Definition 3.2 is still used to select the standard subtableaux. From these we can obtain subwords $w^{(1, k)}, \ldots, w^{(\ell, k)}$ by reading the selected cells columnwise top to bottom, left to right. The definition of index in Definition 3.1 without the terms $\operatorname{diag}(\bar{r}, \overline{r-1})$ is the usual charge of the subwords $w^{(1, k)}, \ldots, w^{(\ell, k)}$. This is due to the fact that the topmost ocurrence of the letter $r$, which we denoted $\bar{r}$, in a weak $k$-tableau in the $(k+1)$-core setting becomes the letter $r$ in the $k$-bounded analogue under the map $\mathfrak{p}$. Note, however, that the selected subwords do depend on $k$ and for small $k$ are usually different from the subwords selected for the usual charge of $T$.

Example 3.5 Take $k=4$ and

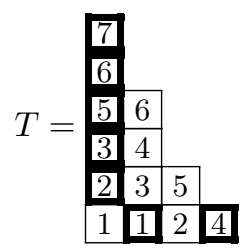

which is the k-bounded analogue of [11. Chapter 2, Example 3.20]. Now we draw the sequence of partitions $\lambda^{(i)}$ and mark the strings in the left columns and the residues of the added boxes as exponents at each step:

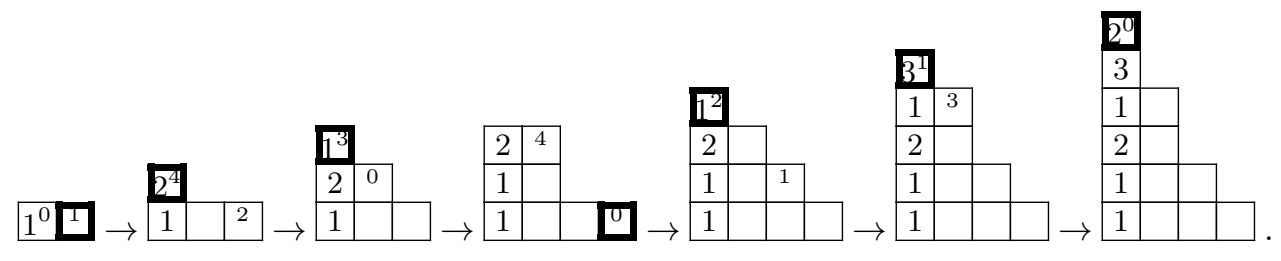

The cells selected for $w^{(1,4)}$ are marked in bold which shows that

$$
w^{(1,4)}=7653214 \text { and } w^{(2,4)}=164352
$$


with charge $\left(w^{(1,4)}\right)+\operatorname{charge}\left(w^{(2,4)}\right)=4+7=11$. Note that the words selected for the usual charge (or for large $k$ ) are different

$$
w^{(1, \infty)}=7326154 \text { and } \quad w^{(2, \infty)}=651432
$$

with charge $\left(w^{(1, \infty)}\right)+\operatorname{charge}\left(w^{(2, \infty)}\right)=4+5=9$.

The term $\operatorname{diag}(\bar{r}, \overline{r-1})$ in the definition of the index in Definition 3.1 can be elegantly expressed in terms of the strings in the partition $\lambda^{(r)}$ (or $\lambda^{(r-1)}$ ). Recall that the letter $\bar{r}$ in a weak $k$-tableaux in the $(k+1)$-core setting becomes the letter $r$ in the $k$-bounded analogue.

Lemma 3.6 Let $T$ be a k-bounded weak k-tableau.

1. If $r$ is east of $r-1$ in $T, \operatorname{diag}(r, r-1)$ is the number of rows strictly between the rows containing $r$ and $r-1$ that are in the same string as the row containing $r$ in $\lambda^{(r)}$.

2. Otherwise, $\operatorname{diag}(r, r-1)$ is the number of rows strictly between the rows containing $r$ and $r-1$ that are in the same string as the row containing $r-1$ in $\lambda^{(r-1)}$.

Proof: Suppose that $r$ is east of $r-1$ in $T$. By definition, $\operatorname{diag}(\bar{r}, \overline{r-1})$ is the number of diagonals of the same residue as the cell containing $\bar{r}$ between $\bar{r}$ and $\overline{r-1}$ in the $(k+1)$-core analogue $\mathfrak{p}^{-1}(T)$ of $T$. In other words, it counts how many multiples of $k+1$ are in the number of cells between $\bar{r}$ and $\overline{r-1}$ when forming a hook between the two cells. Note that by Lemma 2.3 (2) that describes how to pass from a $k$-bounded partition to a $(k+1)$-core using the strings, it suffices to restrict in $T$ to the rows between $r$ and $r-1$. Consider $\lambda^{(r)}$ and let $\tilde{\lambda}^{(r)}$ be the restriction of $\lambda^{(r)}$ to the rows weakly between $r$ and $r-1$ in $T$. The hook distance between $\bar{r}$ and $\overline{r-1}$ in $\mathfrak{p}^{-1}(T)$ is the same as the hook distance in $\mathfrak{p}^{-1}\left(\tilde{\lambda}^{(r)}\right)$ between the corresponding cells. Note that by the definition of strings, the hook distance between the cells at the end of the rows in rows of the same string is always $k+1$. This proves the claim for part (1).

Part (2) can be argued in a similar fashion. Since the strings of the lower cell matter, one uses $\lambda^{(r-1)}$ in this case.

With the definitions and results above, the $k$-charge of Definition 3.1 can be written as

$$
\operatorname{kcharge}(T)=\operatorname{charge}\left(w^{(k)}\right)+\mathcal{O}(T),
$$

where $\mathcal{O}(T)=\sum_{r \geq 1} \mathcal{O}_{r}(T)$ with $\mathcal{O}_{1}(T)=0$ and

$$
\mathcal{O}_{r}=\mathcal{O}_{r-1}+ \begin{cases}\# \text { of rows strictly between rows containing } & \text { if } r \text { east of } r-1, \\ r \text { and } r-1 \text { in same string as } r \text { in } \lambda^{(r)} & \\ -\# \text { of rows strictly between rows containing } & \text { otherwise. } \\ r \text { and } r-1 \text { in same string as } r-1 \text { in } \lambda^{(r-1)} & \end{cases}
$$

Example 3.7 Let us compute $\mathcal{O}_{r}(T)$ for $T$ in Example 3.5. For the first selected standard word we get a contribution to (17) from $r=4$ and $r=5$ of 1 and -1 , respectively (where we filled the rows contributing to (17))

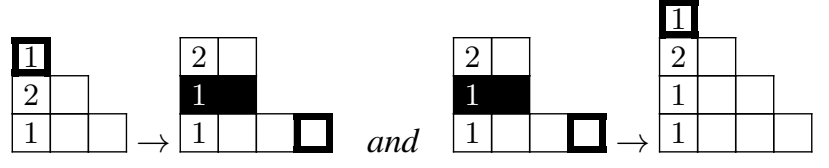


so that $\mathcal{O}_{4}(T)=1$ and all other $\mathcal{O}_{r}(T)=0$, which implies $\mathcal{O}(T)=1$ and $\operatorname{kcharge}(T)=12$.

Theorem 3.8 Let $\lambda, \mu \in \mathcal{P}^{n}$. Then the affine Kostka-Foulkes polynomial is given by

$$
\mathcal{K}_{w_{\lambda}, \mu}(t)=\sum_{T} t^{\mathrm{kcharge}(T)},
$$

where the sum is over all $k$-bounded weak $k$-tableaux of shape $\lambda$ and weight $\mu$ as characterized in Theorem 2.11 with $k$-charge as computed in this section.

Since the recording tableaux of the highest weight KR crystal elements are given in the $k$-bounded language, this can be translated directly to the statistical mechanical formulation of the affine KostkaFoulkes polynomials via [23] as demonstrated in Example 2.12.

\section{References}

[1] L.M. Butler, Subgroup lattices and symmetric functions, Mem. Am. Math. Soc. 112 (1994), no. 539, vi+160 pp.

[2] A. J. Dalal, Several approaches to affine charge, preprint.

[3] A. J. Dalal, J. Morse, The ABC of affine Grassmannians and Hall-Littlewood polynomials, DMTCS proc. AR (2012) 935-946 and Quantum and affine Schubert calculus and Macdonald polynomials, preprint arXiv:1402.1464.

[4] G. Fourier, M. Okado, A. Schilling, Kirillov-Reshetikhin crystals for nonexceptional types, Adv. Math. 222 (2009), no. 3, 1080-1116.

[5] A. M. Garsia, C. Procesi, On certain graded $S_{n}$-modules and the q-Kostka polynomials, Adv. Math. 94 (1992), no. $1,82-138$.

[6] R. Hotta, T. A. Springer, A specialization theorem for certain Weyl group representations and an application to the Green polynomials of unitary groups, Invent. Math. 41 (1977), no. 2, 113-127.

[7] B. Kostant, S. Kumar, The nil Hecke ring and cohomology of G/P for a Kac-Moody group G, Adv. in Math. 62 (1986), no. 3, 187-237.

[8] A.N. Kirillov, N.Y. Reshetikhin, The Bethe ansatz and the combinatorics of Young tableaux, J. Soviet. Math. 41 (1988), 925-955.

[9] T. Lam, Schubert polynomials for the affine Grassmannian, J. Amer. Math. Soc. 21 (2008), no. 1, $259-281$.

[10] T. Lam, L. Lapointe, J. Morse, M. Shimozono, Affine insertion and Piers rules for the affine Grassmannian, Mem. Amer. Math. Soc. 208 (2010), no. 977, xii+82 pp.

[11] T. Lam. L. Lapointe, J. Morse, A. Schilling, M. Shimozono, M. Zabrocki, $k$-Schur functions and affine Schubert calculus, Fields Institute Monographs 33, 2014 (Springer), DOI 10.1007/978-1-4939-0682-6 (arXiv:1301.3569).

[12] L. Lapointe, A. Lascoux, J. Morse, Tableau atoms and a new Macdonald positivity conjecture, Duke Math. J. 116 (2003), no. 1, 103-146.

[13] L. Lapointe, J. Morse, Tableaux on $k+1$-cores, reduced words for affine permutations, and $k$-Schur expansions, J. Combin. Theory Ser. A 112 (2005), no. 1, 44-81. 
[14] L. Lapointe, J. Morse, A k-tableau characterization of k-Schur functions, Adv. Math. 213 (2007), no.1, $183-204$.

[15] L. Lapointe, J. Morse, Quantum Cohomology and the k-Schur Basis, Trans. of the AMS. 360, (2008), no.4, 2021-2040.

[16] L. Lapointe, M.-E. Pinto, Charge on tableaux and the poset of k-shapes, J. Combin. Theory Ser. A 121 (2014), $1-33$.

[17] A. Lascoux, M.-P. Schützenberger, Sur une conjecture de H. O. Foulkes, C. R. Acad. Sci. Paris Sér. A-B 286 (1978), no. 7, A323-A324.

[18] C. Lenart, From Macdonald polynomials to a charge statistic beyond type A, J. Combin. Theory Ser. A 119 (2012), no. 3, 683-712.

[19] C. Lenart, A. Schilling, Crystal energy functions via the charge in types $A$ and $C$, Mathematische Zeitschrift 273(1) (2013) 401-426.

[20] G. Lusztig, Green polynomials and singularities of unipotent classes, Adv. in Math. 42 (1981), no. 2, 169-178.

[21] I. G. Macdonald, Symmetric functions and Hall polynomials. Second edition. Oxford Mathematical Monographs. Oxford Science Publications. The Clarendon Press, Oxford University Press, New York, 1995.

[22] K. Mészáros, private communication, October 2011.

[23] A. Nakayashiki, Y. Yamada, Kostka polynomials and energy functions in solvable lattice models, Selecta Math. (N.S.) 3 (1997), no. 4, 547-599.

[24] D. Peterson, Quantum cohomology of G/P, Lecture notes, M.I.T., Spring 1997.

[25] T. A. Springer, A construction of representations of Weyl groups, Invent. Math. 44 (1978), no. 3, $279-293$. 